\section{Does the risk of lymphoma in patients with RA treated with TNF inhibitors differ according to the histological subtype and the type of TNF inhibitor?}

We read with interest the paper by Mercer et $a l^{1}$ focusing on the risk of lymphoma in patients included in the British Society for Rheumatology Biologics Register for Rheumatoid Arthritis (BSRBR-RA) and treated with tumour necrosis factor inhibitors (TNFi). Based on data from a prospective cohort including $>120000$ patient-years of exposure to TNFi or conventional synthetic disease-modifying drugs (csDMARDs), the authors pointed out that treatment with TNFi in patients with RA was not associated with an increased risk of lymphoma compared with csDMARD treatment. RA is associated with an increased risk of lymphoma, disease activity being the main risk factor of lymphoma occurrence. ${ }^{2}$ Thus, patients with RA treated with TNFi, which have more severely active disease, are supposed to have an increased baseline risk of lymphoma. In this context, the impact of TNFi themselves is unclear. They might decrease the risk of lymphoma over time by controlling activity of the disease. But they also might alter antitumour immunosurveillance and increase the risk of lymphoma as observed in post-transplant lymphoproliferative disease. Thus, large epidemiological studies focusing on the long-term safety of TNFi use as the one presented by Mercer et al are crucial. This important study, in line with results from large cohorts and registers, ${ }^{3} 4$ supports an overall absence of increased risk of lymphoma associated with TNFi compared with csDMARDs, which is very reassuring. However, since there are differences between this 2016 study and a previous one published in 2013 based on the same BSRBR registry, ${ }^{5}$ we have some concerns about the origin of these discrepancies. Moreover, taking advantage of the very large number of patient-years in this study, we would be very interested in having additional analyses concerning the specific risk of diffuse large B cell lymphomas (DLBCLs) and the potential difference of risk of DLBCL according to the type of TNFi; DLBCL being the lymphoma type that is clearly linked to disease activity.

The question of a different impact of the two types of TNFi (monoclonal antibodies compared with the soluble receptor) on the risk of lymphoma is justified by several observations. First, it is recognised that efficacy profile varies depending on the type of TNFi. Etanercept is not effective in inflammatory bowel disease $^{6}$ and is probably less effective than monoclonal in uveitis $^{7}$ and psoriasis. $^{8}$ Second, infectious safety profile differs since the risk of opportunistic infections and of reactivation of tuberculosis has been shown to be higher with monoclonal anti-TNF. $^{9} 10$ These differences support specificities in the mechanism of action (same inhibition of soluble TNF but less inhibition of membrane TNF with the soluble receptor) that might differentially impact the risk of lymphoma. As discussed by Mercer et al, the French registry RATIO has raised the question of a possible differential effect on lymphoma between the monoclonal antibodies and etanercept. ${ }^{11}$ In this prospective pharmacovigilance study, it has been shown that the standardised incidence ratio of lymphoma compared with the general population was 3.7 (95\% CI 2.6 to 5.3) with monoclonal antibodies (that could be expected in a population of severe RA) but does not exceed the risk of the general population in patients treated with etanercept: 0.9 (95\% CI 0.4 to 1.8 ). In the RATIO study, $31 / 38$ patients with lymphoma had been exposed to only one TNFi, which makes the interpretation of the results robust.
In the Japanese cohort SafEty of biologics in Clinical Use in Japanese patients with RhEumatoid arthritis, patients exposed to infliximab had a significant higher risk of lymphoma than patients exposed to etanercept. ${ }^{12}$ Interestingly, in the study presented by Mercer et al, patients exposed to etanercept, whatever the definition of exposure (table 4), are the ones with the lowest crude incidence rate, but the difference between etanercept and csDMARDs was not statistically significant after adjustment. The study presented by Morgan et $a l^{5}$ in 2013 was also based on the BSRBR-RA registry but only focused on the patients treated with etanercept and csDMARDs. As expected, and even if the follow-up was a little bit shorter in the 2013 study, crude incidences were similar between the 2013 and 2016 studies: $0.75 / 1000$ patient-years and $0.70 / 1000$ patient-years in patients treated with etanercept and 1.54/1000 patient-years and 1.60/ 1000 patient-years in patients treated with csDMARDs. However, after adjustment, tendencies were opposite: in the 2016 study, the adjustment led to a disappearance of the lower risk with etanercept versus csDMARDs (HR 1.02 (0.45 to 2.33)) whereas in the 2013 study the lower risk of lymphoma persisted after adjustment (HR 0.51 (0.28 to 0.95)). Certainly, models used for adjustment may differ between the two studies. However, the main risk factor of lymphoma occurrence in patients with RA is disease activity. Patients treated with TNFi are supposed to suffer from a more active disease. Thus, after adjustment, we should expect that the relative lower incidence of lymphoma observed in patients with etanercept should be at least confirmed, which is the case in the 2013 study by Morgan et al, but not in the 2016 study by Mercer et al. Thus, we would be very interested to understand the reason of this discrepancy.

Moreover, we think that the question of histological subtypes deserves additional analyses. Several autoimmune diseases are associated with an increased risk of lymphoma but histological subtype varies with the disease. It is well admitted that the increased risk of lymphoma in patients with RA mainly affects DLBCL. Actually, in the study conducted by Baecklund et $a l^{2}{ }^{13}$ among the 400 cases of lymphoma, about 50\% were DLBCL and they were further characterised as predominantly of the non-germinal centre type. The current study confirmed this result since more than a third of the lymphoma cases were DLBCL. Interestingly, table 3 showed that even not statistically significant, the only subtype of lymphoma numerically more frequent with TNFi than with csDMARDs was DLBCL (HR 1.54 (0.60 to 3.95)). Last, table 4 revealed that the crude incidence of all lymphomas occurrence was lower with etanercept: $0.75 / 1000$ patient-years compared with adalimumab: 0.89; infliximab: 1.02; and csDMARDs: 1.54 . So, we would be very interested to know the HR of DLBCL occurrence between each of the three TNFi individually and csDMARDs and between the different TNFi with each other.

To sum up, we think that data from the BSRBR-RA registry are a great opportunity to raise the question of the risk of lymphoma associated with TNFi exposure in patients with RA and that a specific focus on DLBCL may help to better understand the impact of the different types of TNFi.

\section{Gaetane Nocturne, ${ }^{1}$ Raphaele Seror, ${ }^{2}$ Xavier Mariette ${ }^{1}$}

${ }^{1}$ Hôpitaux Universitaires Paris Sud, Assistance Publique-Hôpitaux de Paris, Le Kremlin Bicêtre, France

2Department of Rheumatology, AP-HP, Hôpital Bicêtre, Le Kremlin Bicêtre, France

Correspondence to Dr Gaetane Nocturne, Hôpitaux Universitaires Paris Sud, Assistance Publique-Hôpitaux de Paris, 78 rue du Général Leclerc, Le KremlinBicêtre 94270, France; gaetanenocturne@gmail.com

Competing interests None declared. 
Provenance and peer review Not commissioned; internally peer reviewed.

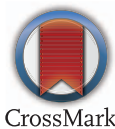

To cite Nocturne G, Seror R, Mariette X. Ann Rheum Dis 2017;76:e3.

Received 20 September 2016

Accepted 25 September 2016

Published Online First 14 October 2016

\section{CLlinked}

http://dx.doi.org/10.1136/annrheumdis-2016-210631

Ann Rheum Dis 2017;76:e3. doi:10.1136/annrheumdis-2016-210566

\section{REFERENCES}

1 Mercer LK, Galloway JB, Lunt M, et al. Risk of lymphoma in patients exposed to antitumour necrosis factor therapy: results from the British Society for Rheumatology Biologics Register for Rheumatoid Arthritis. Ann Rheum Dis 2016; Published Online First: 8 Aug 2016. doi:10.1136/annrheumdis-2016-209389

2 Baecklund E, lliadou A, Askling J, et al. Association of chronic inflammation, not its treatment, with increased lymphoma risk in rheumatoid arthritis. Arthritis Rheum 2006:54:692-701.

3 Lopez-Olivo MA, Tayar JH, Martinez-Lopez JA, et al. Risk of malignancies in patients with rheumatoid arthritis treated with biologic therapy: a meta-analysis. JAMA 2012;308:898-908.
4 Mariette X, Matucci-Cerinic M, Pavelka K, et al. Malignancies associated with tumour necrosis factor inhibitors in registries and prospective observational studies: a systematic review and meta-analysis. Ann Rheum Dis 2011;70:1895-904.

5 Morgan CL, Emery P, Porter D, et al. Treatment of rheumatoid arthritis with etanercept with reference to disease-modifying anti-rheumatic drugs: long-term safety and survival using prospective, observational data. Rheumatology (Oxford) 2014;53:186-94.

6 Sandborn WJ, Hanauer SB, Katz S, et al. Etanercept for active Crohn's disease: a randomized, double-blind, placebo-controlled trial. Gastroenterology 2001;121:1088-94.

7 Guignard S, Gossec L, Salliot C, et al. Efficacy of tumour necrosis factor blockers in reducing uveitis flares in patients with spondylarthropathy: a retrospective study. Ann Rheum Dis 2006;65:1631-4.

8 Zweegers J, Groenewoud JM, van den Reek JM, et al. Comparison of the one and 5 -years effectiveness of adalimumab, etanercept and ustekinumab in psoriasis patients in daily clinical practice: Results from the prospective BioCAPTURE registry. Br J Dermatol 2016. [Epub ahead of print 31 Aug 2016]. doi:10.1111/bjd.15023

9 Salmon-Ceron D, Tubach F, Lortholary O, et al. Drug-specific risk of non-tuberculosis opportunistic infections in patients receiving anti-TNF therapy reported to the 3-year prospective French RATIO registry. Ann Rheum Dis 2011;70:616-23.

10 Furst $D E$. The risk of infections with biologic therapies for rheumatoid arthritis. Semin Arthritis Rheum 2010;39:327-46.

11 Mariette $X$, Tubach F, Bagheri $H$, et al. Lymphoma in patients treated with anti-TNF: results of the 3-year prospective French RATIO registry. Ann Rheum Dis 2010;69:400-8.

12 Harigai $M$, Nanki T, Koike $R$, et al. Risk for malignancy in rheumatoid arthritis patients treated with biological disease-modifying antirheumatic drugs compared to the general population: a nationwide cohort study in Japan. Mod Rheumatol 2016;26:642-50.

13 Baecklund E, Natkunam Y, Backlin C, et al. Expression of the human germinal-centre-associated lymphoma protein in diffuse large B-cell lymphomas in patients with rheumatoid arthritis. Br J Haematol 2008;141:69-72. 